\title{
Role of preoperative electrocardiography in predicting cardiovascular complications in proximal femur surgery
}

\author{
Regayip Zehir $\mathrm{MD}^{1}$, Sinan Zehir $\mathrm{MD}^{2}$, Gonenc Kocabay $\mathrm{MD}^{3}$
}

\begin{abstract}
R Zehir, S Zehir, G Kocabay. Role of preoperative electrocardiography in predicting cardiovascular complications in proximal femur surgery. Curr Res Cardiol 2015;2(4):171-174.
\end{abstract}

OBJECTIVE: To evaluate whether a complete analysis of electrocardiograms (ECGs) taken before hip fracture surgery could provide prognostic data regarding in-hospital perioperative cardiovascular complications (PCVCs).

METHODS: Elderly patients who underwent surgery using a proximal femoral nail due to peritrochanteric fracture between 2009 and 2013 were retrospectively evaluated. Patients' 12-lead preoperative ECGs were analyzed and corrected QT intervals (QTcs) were calculated. Following analysis, ECGs with atrial fibrillation, left or right bundle branch block, ventricular premature beats, $Q$-waves, ventricular strain, sinus bradycardia or tachycardia were designated as abnormal. The relationship between observed ECG abnormalities and calculated QTc interval, and the development of in-hospital PCVC was evaluated.

E ectrocardiography (ECG) is commonly performed on patients scheduled for noncardiac surgery as part of risk evaluation. Whereas ECG has been shown to be ineffective in determining perioperative cardiac risk in low-risk surgeries, routine ECG is recommended before intermediate-risk surgeries where there are $\geq 1$ clinical risk factors or an existing vascular disease in the patient history (1). Cardiac complications that lead to mortality and morbidity develop in approximately $5 \%$ of orthopedic surgeries in patients who are at intermediate risk in terms of perioperative cardiovascular complications (PCVCs) (2). Because most cardiac complications are treatable, prediction and/or early diagnosis are vital.

Currently, proximal femur fractures comprise $30 \%$ of all fractures presenting to hospital. Increases in the elderly population and, thus, increases in senile osteoporosis and post-menopausal status has led to an increase in incidences of proximal femur fractures requiring priority treatment (3). A significant proportion of patients with hip fractures have cardiovascular diseases. Hip fractures are an important cause of morbidity and mortality among elderly patients. Mortality in the first three months following a hip fracture has been found to be $7.95 \%$ for elderly men and $5.75 \%$ for elderly women (4). Although reduction of perioperative and postoperative mortality are the priority target of the surgical team, these are closely linked to the accurate assessment of the patient's operative and cardiac risk.

While PCVC risk evaluation in patients who are undergoing vascular operations has been widely studied in the literature, the incidence and predictors of this risk have not sufficiently been explored in patients with hip fractures, which are increasingly more common. Therefore, we retrospectively evaluated whether a complete analysis of
RESULTS: In total, 190 male and 232 female patients were included in the study. Two hundred nineteen $(51.9 \%)$ patients had a fractured right hip and $203(48.1 \%)$ had a fractured left hip. ECGs of 133 patients were abnormal. Seventeen patients $(4.02 \%)$ died and 62 patients $(14.6 \%)$ developed PCVCs. There was a statistically significant increase $(\mathrm{P}=0.0183)$ in PCVC as perioperative cardiac risk factors increased. Of the abnormal ECG changes, only atrial fibrillation, paced rhythm and ventricular strain were found to be related to PCVCs $(\mathrm{P}<0.001, \mathrm{P}<0.03$ and $\mathrm{P}<0.001$, respectively). It was found that PCVCs increased significantly as the $\mathrm{QT}$ interval increased $(\mathrm{P}<0.0001)$. QTc prolongation was correlated with PCVC.

CONCLUSION: ECG changes before hip fracture surgery, especially QTc prolongation, was found to be closely linked to PCVCs.

Key Words: Electrocardiogram; Perioperative cardiovascular complications; Proximal femur fracture

ECGs obtained before hip fracture surgery could provide prognostic data regarding in-hospital PCVCs.

\section{METHODS}

Elderly patients who underwent surgery using a proximal femoral nail due to peritrochanteric fracture between 2009 and 2013 were retrospectively evaluated. Emergency cases and patients who were not expected to live after $24 \mathrm{~h}$ without surgery due to multiple traumas were excluded. For each patient, preoperative risk factors mentioned in the revised cardiac risk index (ie, ischemic heart disease, congestive heart failure, history of cerebrovascular accident, diabetes mellitus requiring insulin treatment, preoperative kidney failure) (5), preoperative medications, patient characteristics and intraoperative techniques that could affect morbidity and mortality were retrospectively evaluated. Twelve-lead ECGs of patients recorded at $25 \mathrm{~mm} / \mathrm{s}$ at the time of entry to the hospital were analyzed by two blinded researchers. All ECGs were analyzed for Minnesota classification, QRS duration, QT interval and left ventricular hypertrophy (6). QT interval was corrected for the heart rate using Bazett's formula (7). For corrected QT interval (QTc), interobserver and intraobserver variability was $8 \%$ and $7 \%$, respectively. Following analysis, ECGs with atrial fibrillation (AF), left or right bundle branch block, ventricular premature beats, $\mathrm{Q}$-waves, ventricular strain, sinus bradycardia or tachycardia were defined as abnormal.

PCVC was defined as the occurrence of severe arrhythmia requiring treatment, cardiac death (death caused by acute myocardial infarction, serious cardiac arrhythmia or deaths occurring suddenly without other explanation), acute cardiac failure, acute coronary syndrome (nonfatal acute myocardial infarction or unstable angina),

${ }^{1}$ Dr Siyami Ersek Heart and Research Hospital, Istanbul; ${ }^{2}$ Hitit University, Faculty Of Medicine, Department Of Orthopedics $\mathcal{B}$ Traumatology, Çorum;

${ }^{3}$ Kartal Kosuyolu Heart and Research Hospital, Istanbul, Turkey

Correspondence: Dr Gonenc Kocabay, Department of Cardiac, Thoracic and Vascular Sciences, University of Padua, Centro Gallucci, Via Giustiniani 2 , 35128, Padua, Italy. Telephone 39-049-8218642, fax 39-049-8761764, e-mail gonenckocabay@yahoo.com 
TABLE 1

Perioperative cardiovascular complications (PCVCs)

\begin{tabular}{|c|c|c|c|c|c|c|c|c|c|c|}
\hline & \multicolumn{9}{|c|}{ PCVCs, $n$} & \multirow[b]{2}{*}{ Total } \\
\hline & $\begin{array}{c}\text { Cardiac } \\
\text { death }\end{array}$ & $\begin{array}{c}\text { Nonfatal } \\
\text { cardiac arrest }\end{array}$ & $\begin{array}{l}\text { Pulmonary } \\
\text { embolism }\end{array}$ & $\begin{array}{l}\text { Acute coronary } \\
\text { syndrome }\end{array}$ & Stroke & $\begin{array}{l}\text { Acute cardiac } \\
\text { failure }\end{array}$ & $\begin{array}{c}\text { Severe } \\
\text { arrhythmia }\end{array}$ & None & DVT & \\
\hline \multicolumn{11}{|c|}{ Electrocardiogram } \\
\hline Abnormal & 11 & 4 & 1 & 2 & 3 & 8 & 6 & 94 & 4 & 133 \\
\hline Total & 17 & 5 & 3 & 5 & 5 & 13 & 19 & 343 & 12 & 422 \\
\hline \multicolumn{11}{|c|}{ Perioperative cardiac risk factors } \\
\hline 1 & 0 & 1 & 2 & 0 & 2 & 1 & 2 & 129 & 3 & 140 \\
\hline 2 & 7 & 2 & 0 & 2 & 2 & 4 & 6 & 112 & 3 & 138 \\
\hline$\geq 3$ & 9 & 2 & 1 & 2 & 1 & 7 & 9 & 18 & 4 & 53 \\
\hline Total & 17 & 5 & 3 & 5 & 5 & 13 & 19 & 343 & 12 & 422 \\
\hline \multicolumn{11}{|c|}{ Fracture type } \\
\hline A2.1 & 6 & 1 & 1 & 2 & 2 & 3 & 4 & 66 & 3 & 88 \\
\hline A3.3 & 0 & 0 & 1 & 0 & 0 & 0 & 1 & 5 & 0 & 7 \\
\hline Total & 17 & 5 & 3 & 5 & 5 & 13 & 19 & 343 & 12 & 422 \\
\hline \multicolumn{11}{|c|}{ American Society of Anesthesiologists score } \\
\hline 2 & 1 & 0 & 0 & 1 & 0 & 1 & 1 & 48 & 0 & 52 \\
\hline 3 & 6 & 2 & 3 & 2 & 3 & 8 & 8 & 171 & 10 & 213 \\
\hline 4 & 10 & 3 & 0 & 2 & 2 & 4 & 10 & 124 & 2 & 157 \\
\hline Total & 17 & 5 & 3 & 5 & 5 & 13 & 19 & 343 & 12 & 422 \\
\hline
\end{tabular}

DVT Deep vein thrombosis

acute pulmonary thromboembolism, nonfatal cardiac arrest and cardioembolic stroke.

\section{Surgical method}

After patients' written consent was received and optimal conditions were provided for all patients, surgical intervention was performed with the patient lying on his/her back under the scope according to standard protocols described by the producers. Prophylactic first-generation cephalosporins were administered to all patients. In the preoperative period, patients were started on $0.4 \mathrm{mg}$ daily dose of subcutaneous low molecular weight heparin injections (enoxaparin) to prevent deep vein thrombosis. Postoperatively, all patients wore antiembolism compression hose. After the operation, patients were encouraged to walk putting partial pressure on the operated extremity.

\section{Statistical analysis}

Statistical analysis was performed using $\chi^{2}$, Kaplan-Meier estimates and Spearman correlation. SPSS version 21.0 (IBM Corporation Inc, USA) for Windows (Microsoft Corporation, USA) package was used for all analyses. $\mathrm{P}<0.05$ was considered to be statistically significant.

\section{RESULTS}

In total, 190 male and 232 female patients were included in the study. Of these, 219 (51.9\%) had a right hip fracture and 203 (48.1\%) had a left hip fracture. Mean $( \pm$ SD) age was $77.68 \pm 7.67$ years, mean hospital stay was $10.66 \pm 4.02$ days and mean preoperative period was $3.48 \pm 1.99$ days. Thirty-one (7.34\%) patients received general anesthetic and $391(92.66 \%)$ patients received regional anesthesia. ECGs of 133 patients were abnormal. Mean QTc interval was found to be $426.45 \pm 16.07 \mathrm{~ms}$. Of the 422 patients, $101 \mathrm{did}$ not have any preoperative cardiac risk factors and $25 \mathrm{had} \geq 3$. Seventeen patients $(4.02 \%)$ died and $62(14.6 \%)$ developed PCVCs. Twelve patients developed superficial surgical site infection and 14 patients developed urinary tract infection. Treatment for infection was provided through appropriate care and antibiotics. Two patients experienced deep surgical site infection; despite debridement and antibiotics, infection could not be controlled and, thus, the implant was removed. Treatment for infection and the fracture was performed with an external fixator device. Three patients underwent reoperation due to nonunion and 13 due to cut out.

No statistically significant link between PCVC and sex, fracture type or fracture side was found $(\mathrm{P}>0.05)$ (Table 1). Median patient age (78 years) was used as a cut-off point; it was determined that there was a statisictally significant increase in PCVCs in patients older than 78 years $(\mathrm{P}<0.0001)$. Furthermore, increased preoperative cardiac risk factors were associated with increased PCVCs $(\mathrm{P}=0.018)$.

When patients were evaluated in terms of the methods of anesthesia and American Society of Anesthesiologists (ASA) physical status classification system scores, it was seen that while method of anesthesia did not significantly impact PCVCs, there was a significant relationship between higher ASA score (scores of 3 and 4$)$ and PCVCs $(\mathrm{P}<0.001)$.

Of the abnormal ECG changes, only AF, paced rhythm and ventricular strain were found to be related to PCVCs $(\mathrm{P}<0.001, \mathrm{P}<0.03$ and $\mathrm{P}<0.001$, respectively) (Table 2). It was found that as QT interval increased, PCVCs increased statistically $(\mathrm{P}<0.0001)$. In patients with abnormal ECGs and QT intervals above the median limit of $426.45 \mathrm{~ms}$, PCVC ratio was found to be 1.8 times higher. The prolongation of the QT interval was found to be correlated to PCVC $(\mathrm{r}=0.365 ; \mathrm{P}<0.001)$.

\section{DISCUSSION}

Results of the present study have shown that standard preoperative ECGs can predict postoperative PCVCs that may develop following proximal femur fracture surgeries (which are classified as intermediaterisk surgery for cardiac-related events). Preoperative cardiac risk factors are closely related to perioperative cardiac mortality and morbidity in elderly femur fracture patients. In addition, a prolonged QTc interval, which can easily be calculated from an ECG, is an independent risk indicator for PCVCs that may develop following proximal femur fracture surgeries. Moreover, patients with abnormal ECGs and/or prolonged QTc intervals but no other history of preoperative cardiac 
risk factors represent an important group because of the potential for risk-reduction intervention.

As the human lifespan increases, incidence of hip fractures increases. The one-year mortality rate in hip fracture patients is between $12 \%$ and $36 \%$, and is highest during the first month (8). It has been proven that cardiovascular comorbidity has an important effect on patient survival. Moreover, it is known that important postoperative complications that may affect lifespan are closely linked to preoperative cardiac risk factors (9). Although early surgical intervention reduces mortality in patients with multiple cardiac risk factors, determining cardiac risk accurately and quickly, and preventing PCVCs may reduce additional mortality and morbidity. However, especially in elderly patients, in addition to patient's medical histories, unnecessary, expensive and time-consuming tests conducted to determine preoperative cardiac risk may put patients in unexpected risk by delaying surgery.

There are various views on the role of routine preoperative ECGs in predicting PCVCs before surgical procedures with intermediate or high cardiac risk such as proximal femur surgeries. Incidence of abnormal ECGs are higher in elderly patients due to increasing morbidity. In a large patient series $(n=23,036)$, preoperative ECG was found to predict early cardiovascular mortality in addition to cardiac risk factors (10). Each abnormal ECG change provided similar results, and even after age was included in the regression model, preoperative ECG changes remained meaningful. Similarly, Van Klei et al (11) reported that the bundle branch block was related to postoperative myocardial infarction. Correll et al (12) reported an association between abnormal ECG, postoperative cardiovascular complications and death due to all causes. In contrast, in a study involving patients older than 70 years, the role of preoperative ECG in predicting perioperative cardiac complications was shown to be small (13). However, PCVC was limited in this study and 22 ECG variabilities were analyzed, which may have decreased specificity. Moreover, in this study, QTc intervals were not considered. In our study, QTc prolongation predicted PCVCs. None of the patients used any medication before inclusion that may have affected QT interval. Furthermore, the prophylactic cephalosporin antibiotics used in the present study do not have any reported effect on QT interval.

ASA score is a reliable assessment of physical status of an individual before the surgical procedure, and is accepted as a simple and effective tool to predict surgical short- and long-term outcomes. Higher ASA scores indicate significant perioperative morbidity. We found significant correlation between ASA grade and PCVCs, as expected. Severe systemic disturbances (ASA grade 3) and extreme systemic disorders that have already become an eminent threat to life (ASA grade 4) may be the cause of abnormal ECGs, especially altered QT interval. Moreover, Yeoh et al (14) concluded that higher ASA grade was associated with delay in surgery, longer hospital stay and higher 30-day mortality in elderly patients with femoral neck fracture.

Perioperative AF is an independent predictor of worse early and late outcomes after coronary artery bypass surgery. It also increases the risk for ischemic stroke after noncardiac surgery (15). Pace-induced mechanical dyssynchrony is an important factor that affects cardiac output. Paced ECG complicates the probable diagnosis of cardiac arrhythmias. Moreover, pacemaker malfunction not clearly diagnosed on ECGs can impair effective cardiac functions. Ventricular strain pattern on ECG is associated with stroke, heart failure development and myocardial infarction in large population studies. Strain pattern is accepted as electrocardiographic biomarker and its presence is suggested to guide management of individual patients (16).

Prolongated QTC interval is a significant predictor of cardiacrelated deaths. In the general population, it is related to increased 10 -year ischemic heart disease risk and sudden cardiac death risk $(17-20)$. Hanci et al (21) reported that in patients with prolongated QTc interval, more arrhythmia was observed during anesthesia and postoperatively. The present study has shown that in patients
TABLE 2

Predictive value of QTc interval

\begin{tabular}{|c|c|c|c|}
\hline & \multicolumn{2}{|c|}{ QTc, ms } & \multirow[b]{2}{*}{$\mathbf{P}$} \\
\hline & $<426.45$ & $\geq 426.45$ & \\
\hline \multicolumn{4}{|c|}{ Perioperative cardiovascular complications } \\
\hline Cardiac death & 5 & 12 & 0.001 \\
\hline Nonfatal cardiac arrest & 2 & 3 & 0.001 \\
\hline Pulmonary embolism & 1 & 2 & 0.001 \\
\hline Acute coronary syndrome & 2 & 3 & 0.001 \\
\hline Stroke & 1 & 4 & 0.001 \\
\hline Acute cardiac failure & 4 & 9 & 0.001 \\
\hline Severe arrhythmia & 13 & 6 & 0.001 \\
\hline Deep vein thrombosis & 5 & 7 & 0.13 \\
\hline \multicolumn{4}{|l|}{ Fracture type } \\
\hline A2.1 & 51 & 37 & 0.06 \\
\hline A2.2 & 111 & 69 & 0.06 \\
\hline A2.3 & 75 & 49 & 0.07 \\
\hline A3.1 & 11 & 5 & 0.16 \\
\hline A3.2 & 3 & 4 & 0.23 \\
\hline A3.3 & 6 & 1 & 0.39 \\
\hline \multicolumn{4}{|c|}{ American Society of Anesthesiologists score } \\
\hline 2 & 36 & 16 & 0.06 \\
\hline 3 & 129 & 84 & 0.07 \\
\hline 4 & 92 & 65 & 0.06 \\
\hline Abnormal electrocardiogram & 46 & 87 & 0.001 \\
\hline \multicolumn{4}{|c|}{ Perioperative cardiac risk factors } \\
\hline 1 & 92 & 73 & 0.08 \\
\hline 2 & 91 & 40 & 0.04 \\
\hline$\geq 3$ & 15 & 10 & 0.02 \\
\hline
\end{tabular}

Data presented as $n$ unless otherwise indicated. QTC Correct QT interval

who underwent nonvascular surgery, preoperative prolongated QTC interval was related to perioperative cardiovascular complications independent of all other factors.

\section{Limitations}

Our study was based on the data derived from only one medical centre. Patients were evaluated retrospectively from archived data. In the study, only in-hospital complications were considered. Early and later findings outside of the hospital were not included. There is a need for a prospective study where long-term follow-up is conducted on a larger group of patients.

\section{CONCLUSION}

ECG changes and, in particular, the QTc interval, before hip fracture surgery has been determined to be closely linked to PCVCs. Moreover, these ECG findings may change the ASA score, which is a predictor of all possible complications. QTc derived from preoperative ECG has additional benefits in predicting risk of complications in elderly patients with hip fracture. Such patients with prolongated QTc interval may benefit from monitoring for perioperative problems by a multidisciplinary team consisting of an orthopedist, anesthesiologist, geriatrician and cardiologist.

DISCLOSURES: The authors have no financial relationships or conflicts of interest to declare.

\section{REFERENCES}

1. Kristensen SD, Knuuti J, Saraste A, et al. 2014 ESC/ESA Guidelines on noncardiac surgery: Cardiovascular assessment and management: The Joint Task Force on Non-cardiac Surgery: Cardiovascular assessment and management of the European Society of Cardiology (ESC) and the European Society of Anaesthesiology (ESA). Eur J Anaesthesiol 2014 [Epub ahead of print]. 
2. Modesti PA, Simonetti I, Olivo G. Perioperative myocardial infarction in non-cardiac surgery. Pathophysiology and clinical implications. Intern Emerg Med 2006;1:177-86.

3. Kannus P, Parkkari J, Sievänen H, et al. Epidemiology of hip fractures. Bone 1996;18(Suppl 1):57S.

4. Haentjens P, Magaziner J, Colón-Emeric CS, et al. Meta-analysis: Excess mortality after hip fracture among older women and men. Ann Intern Med 2010;152:380.

5. Lee TH, Marcantonio ER, Mangione CM, et al. Derivation and prospective validation of a simple index for prediction of cardiac risk of major noncardiac surgery. Circulation 1999;100:1043-9.

6. Blackburn H, Keys A, Simonson E, et al. The electrocardiogram in population studies. Circulation 1960;21:1160-75.

7. Hannan PJ, Crow RS. Concerning the units for the QT interval corrected by Bazett's formula. Circulation 1997;96:3799.

8. González-Rozas M, Pérez-Castrillón JL, González-Sagrado M, et al. Risk of mortality and predisposing factors after osteoporotic hip fracture: A one-year follow-up study. Aging Clin Exp Res 2012;24:181-7.

9. Muraki S, Yamamoto S, Ishibashi H, et al. Factors associated with mortality following hip fracture in Japan. J Bone Miner Metab 2006;24:100-4.

10. Noordzij PG, Boersma E, Bax JJ, et al. Prognostic value of routine preoperative electrocardiography in patients undergoing noncardiac surgery. Am J Cardiol 2006;1103-6.

11. Van Klei WA, Bryson GL, Yang H, et al. The value of routine preoperative electrocardiography in predicting myocardial infarction after noncardiac surgery. Ann Surg 2007;246:165-70.

12. Correll DJ, Hepner DL, Chang C, et al. Preoperative electrocardiograms: Patient factors predictive of abnormalities. Anesthesiology 2009;110:1217-22.
13. Liu LL, Dzankic S, Leung JM. Preoperative electrocardiogram abnormalities do not predict postoperative cardiac complications in geriatric surgical patients. J Am Geriatr Soc 2002;50:1186-91.

14. Yeoh CJ, Fazal MA. ASA grade and elderly patients with femoral neck fracture. Geriatr Orthop Surg Rehabil 2014;5:195-9.

15. Pullicino P. Incident perioperative AF increased risk for ischemic stroke at 1 year, with greater risk after noncardiac surgery. Ann Intern Med 2015;20:162.

16. Schocken DD. Electrocardiographic left ventricular strain pattern: Everything old is new again. J Electrocardiol 2014;47:595-8.

17. De Bruyne MC, Hoes AW, Kors JA, et al. Prolonged QT interval predicts cardiac and all-cause mortality in the elderly. The Rotterdam Study. Eur Heart J 1999;20:278-84.

18. Karjalainen J, Reunanen A, Ristola P, Viitasalo M. QT interval ASA cardiac risk factor in a middle aged population. Heart 1997;77:543-8.

19. Crow RS, Hannan PJ, Folsom AR. Prognostic significance of corrected QT and corrected JT interval for incident coronary heart disease in a general population sample stratified by presence or absence of wide QRS complex: The ARIC study with 13 years of follow-up. Circulation 2003;108:1985-9.

20. Lee J, Yoo K, Oh Y, et al. Relationship between resting electrocardiographic parameters and estimated 10-year risk for coronary heart disease in healthy adults in the USA. Ann Noninvas Electrocardiol 2010;15:315-20.

21. Hanci V, Yurtlu S, Aydin M, et al. Preoperative abnormal P and QTc dispersion intervals in patients with metabolic syndrome. Anesth Analg 2011;112:824-7. 